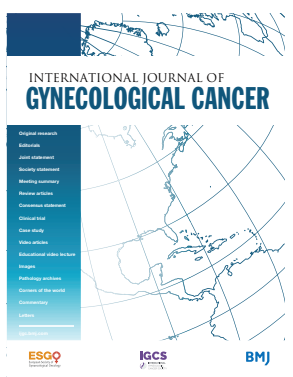

\title{
Placenta increta mimicking placental site trophoblastic tumor
}

\author{
Mitchell Clark \\ Gynecologic Oncology, Yale School of Medicine, New Haven, Connecticut, USA \\ Nairi Tchrakian \\ Toronto General Hospital, Toronto, Ontario, Canada \\ Blaise Clarke \\ Laboratory Medicine and Pathobiology, University of Toronto, Toronto, Ontario, Canada \\ Ur Metser \\ Medical Imaging, University of Toronto Faculty of Medicine, Toronto, Ontario, Canada \\ Geneviève Bouchard-Fortier \\ Gynecologic Oncology, Princess Margaret Hospital Cancer Centre, Toronto, Ontario, Canada
}

\section{Correspondence to} Dr Geneviève Bouchard-Fortier, Gynecologic Oncology, Princess Margaret Hospital Cancer Centre, Toronto, ON M5G 2C1, Canada; genevieve.bouchardfortier@uhn.ca

Accepted 9 August 2021
Check for updates

(C) IGCS and ESGO 2021. № commercial re-use. See rights and permissions. Published by BMJ.

To cite: Clark M, Tchrakian N, Clarke B, et al. Int J Gynecol Cancer 2021;31:1481-1485.

\section{CASE PRESENTATION}

A 35-year-old woman $G_{5} P_{2}$ was referred to the gynecologic oncology service for evaluation and management of a large cornual mass on MRI. She was otherwise healthy with no previous surgery or comorbidities. She had two uncomplicated vaginal deliveries and two previous miscarriages. Four months prior to presentation, the patient underwent a first trimester ultrasound at 8 weeks' gestation, demonstrating a single intrauterine gestational sac measuring $6^{+0}$ weeks gestation with two small sub-chorionic hemorrhages and no embryo. Her serum human chorionic gonadotropin (HCG) level was 65083 IU/L. A follow-up ultrasound was performed 2 weeks later and confirmed miscarriage with no growth and absence of cardiac activity.

The patient was given $800 \mu \mathrm{g}$ misoprostol and required a second dose for retained products of conception on repeat imaging. She did not experience any additional bleeding after the second dose of misoprostol. Repeat ultrasound demonstrated a new cornual mass with myometrial invasion concerning for an arteriovenous malformation. She had persistent low serum HCG levels over 2 weeks at $89 \mathrm{IU} / \mathrm{L}$, $93 \mathrm{IU} / \mathrm{L}$, and $74 \mathrm{IU} / \mathrm{L}$. She was referred to the gynecologic oncologist for further evaluation. She reported persistent mild right-lower quadrant pain and fullness. She had no vaginal bleeding. A pelvic MRI was obtained to better characterize the suspicious uterine lesion.

\section{DR METSER}

This mass was further characterized by MRI demonstrating a heterogeneous, intermediate-high T2 signal, enhancing $5.2 \times 3.8 \mathrm{~cm}$ right cornual mass with extension into the myometrium and increased vascularity.
The mass contained dilated serpiginous vessels measuring up to $14 \mathrm{~mm}$ caliber, demonstrating arterial phase enhancement and early venous drainage into prominent right-sided adnexal and gonadal veins. There were scattered interposed cystic and possibly hemorrhagic regions. The remainder of the myometrium was diffusely heterogeneous with generalized increased vascularization (Figure 1).

CT scan of the chest, abdomen, and pelvis showed no evidence of metastatic disease. Given the persistent low level serum HCG and MRI findings, our differential diagnosis included a placenta increta, intermediate trophoblastic diseases such as placental site trophoblastic tumor or epithelioid trophoblastic tumor, or an arteriovenous malformation.

\section{DR METSER}

How can we differentiate between placental site trophoblastic disease, placenta increta and arteriovenous malformation on imaging? Differentiating between these entities can be difficult as imaging characteristics often overlap. MRI has been studied and characteristic findings of arteriovenous malformations can include serpentine flowrelated signal voids in the endometrium, uterine wall as well as parametrium on T1- and T2-weighted images. Unfortunately, these same appearances can be appreciated in the presence of gestational trophoblastic disease, with the distinguishing difference being the presence or absence of an intermediate T2 signal intensity mass on MRI and human chorionic gonadotropin. ${ }^{1}$ Arteriovenous malformations may also have a 'cluster of grapes' appearance given the presence of extensive cystic spaces without a true solid component. There are no large series describing the classic radiographic appearances of retained placenta 


\section{Case study}

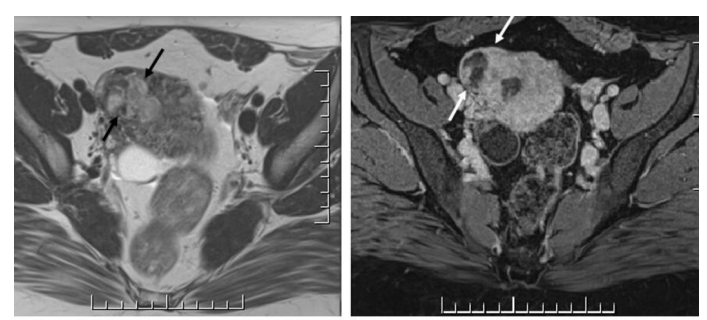

Figure 1 Pelvic MRI: Axial T2-weight image (left) and axial post-gadolinium fat-saturated T1 image (right) show a heterogeneous $5.2 \times 3.8 \mathrm{~cm}$ intermediate-high T2 signal intensity mass extending into and expanding the right cornua, with near complete full thickness invasion of the myometrium (arrows). MR angiography demonstrates asymmetric increased vascularity within the right fundal myometrium and arteriovenous shunting.

increta/accreta. Limited data regarding this rare condition are focused on intra-partum diagnosis. A series of 14 cases suspicious for retained placenta accreta found the presence of a hyperintense area on T2-weighted images with transient early enhancement and the absence of delayed strong enhancement were predictors of placenta accreta. ${ }^{2}$

The patient had completed her childbearing and underwent an uncomplicated open total abdominal hysterectomy and bilateral salpingectomy. Intra-operatively, she was found to have a $5 \mathrm{~cm}$ right cornual vascular mass, intact serosa, and otherwise normal fallopian tubes and ovaries (Figure 2).

\section{Dr Tchrakian: Can you describe the pathologic findings that led to the diagnosis of placenta increta after missed abortion and discuss the role of ancillary studies?}

Macroscopic assessment of the specimen showed a normal size uterus without evidence of uterine serosal breach. On sectioning, a polypoid red-brown mass was identified, centered in the right cornual region, $4.5 \mathrm{~cm}$ in maximum dimension. The mass demonstrated deep extension into the myometrium, to within $0.1 \mathrm{~cm}$ of the serosal surface of the uterus. The lower uterine segment and cervix were not involved. The entire mass was submitted for histologic evaluation. The sections showed chorionic villi extending into the myometrium without intervening decidua, diagnostic of placenta increta. The clinical suspicion of a neoplastic process was noted at the time of reporting; however, histologic features of placental-site trophoblastic tumors including sheets of intermediate trophoblast infiltrating myometrium in a diffuse fashion with associated eosinophilic/fibrinoid material, frequently with accompanying necrosis, were not seen.
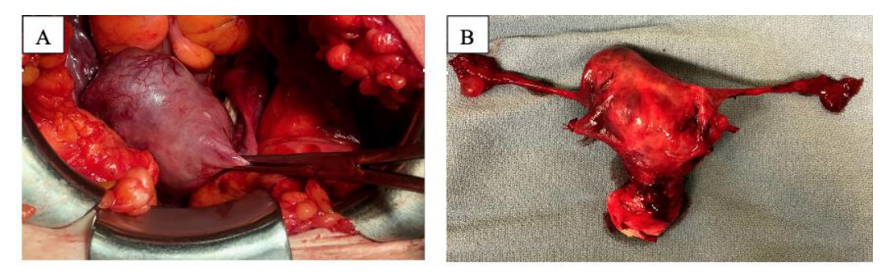

Figure 2 (A) Uterus in situ with $5 \mathrm{~cm}$ right cornual vascular mass. (B) Surgical specimen including uterus, cervix and bilateral tubes with right cornual mass, $8.0 \times 6.0 \times 4.4 \mathrm{~cm}$, $114 \mathrm{~g}$.
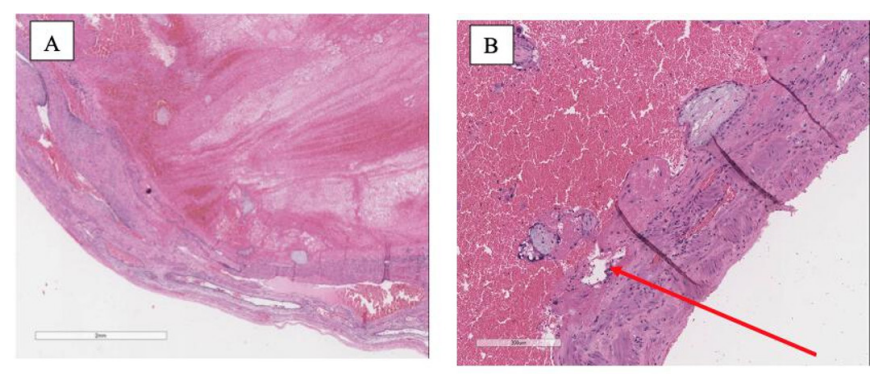

Figure 3 (A) Low power hematoxylin and eosin image of markedly thinned myometrial wall with overlying hemorrhage and scattered chorionic villi. (B) High power image of markedly thinned myometrium bearing immediately adjacent chorionic villi with associated Nitabuch fibrin (arrow) without intervening decidua, in keeping with placenta increta.

Although some cases of placenta accreta may be subtle (or even occult), the severe myometrial thinning in this case of placenta increta was well developed (Figure 3A,B). Some sections taken from the periphery of the abnormality showed foci of near-normal implantation (placenta with intervening endometrium) (Figure 4). The majority of the implantation site, however, consisted of placental tissue separated from underlying myometrium with only a strip of (Nitabuch's) fibrin in between, devoid of a decidual layer (Figure 5A,B). At the point of deepest involvement, the uterine serosa was $<1 \mathrm{~mm}$ clear, hence the designation of 'near percreta' in the pathology report.

The chorionic villi were largely viable with normal configuration (relatively uniform size and shape, without significant enlargement or hydropic change). Focally, however, some non-viable villi were seen which appeared enlarged with irregular contours, raising the possibility of a partial hydatidiform mole (Figure 6A). Immunostaining for p57 showed intact staining in villous stromal cells and cytotrophoblast, thus excluding a complete hydatidiform mole (Figure 6B). Cytogenetic/ploidy studies were attempted to exclude

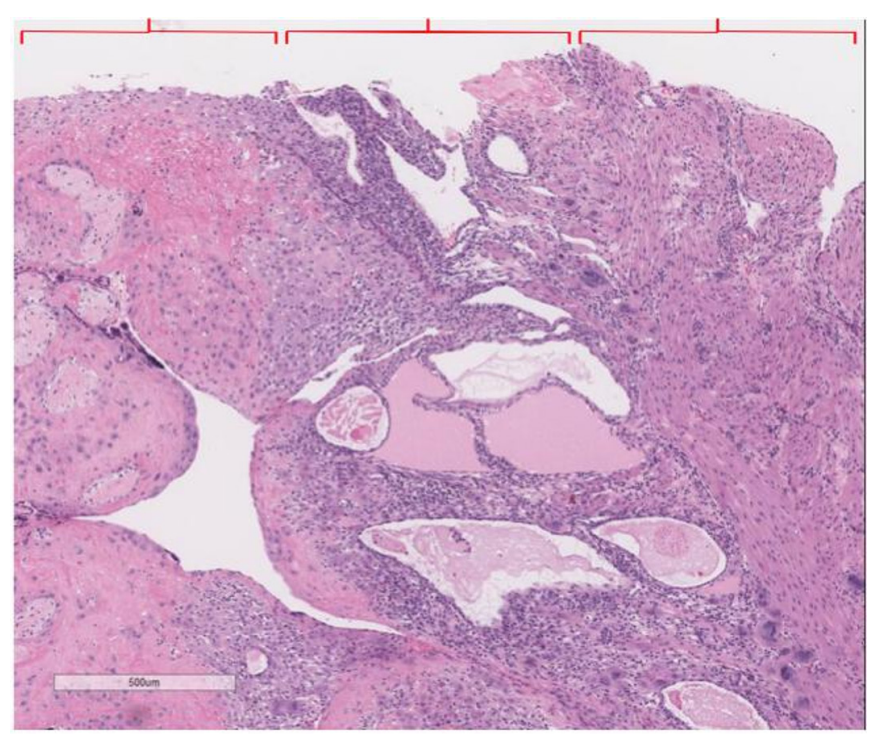

Figure 4 Medium power image of a peripheral section showing relatively normal implantation-chorionic villi (left) are separated from myometrium (right) by endometrium (center). 

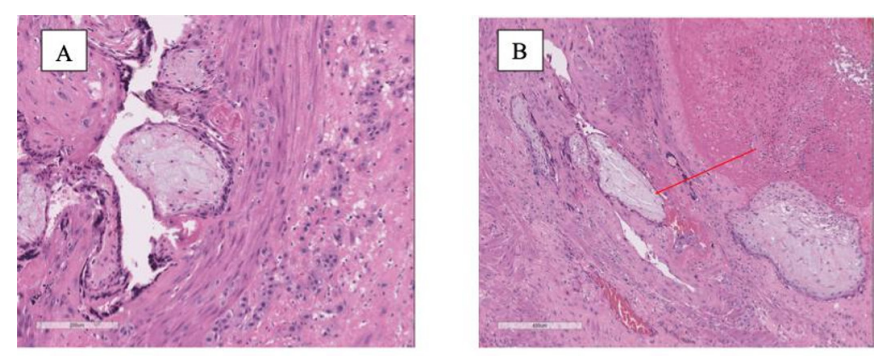

Figure 5 A) High power image of chorionic villi directly opposed to myometrium without intervening decidua. Invasive trophoblast is seen to infiltrate through the myometrium (right side of image). (B) Medium power image of placenta increta demonstrating a chorionic villus within a dilated myometrial vascular space (arrow).

partial hydatidiform mole but were unsuccessful due to intermingling of placental and maternal tissues. The index of suspicion for partial hydatidiform mole was low due to the fact that other features including trophoblastic proliferation or pseudo-inclusions were not seen.

Lastly, with regard to the role of ancillary studies in these cases, the diagnosis of placenta accreta/increta/percreta is one which is made primarily on morphologic findings. ${ }^{3}$ The gross features are of paramount importance, and close inspection for serosal breach, evidence of prior cesarian section scar and abnormal placental location (such as placenta previa) as well as thorough dissection to evaluate the depth of placental penetration into myometrium are warranted. ${ }^{4}$ Judicious sampling for histology should reveal the cardinal feature of all these disorders - namely, implantation of placenta into myometrium without intervening decidua — which is usually evident on routine H\&E [hematoxylin and eosin] staining. In challenging cases, however, immunohistochemistry for vimentin, cytokeratin and desmin may help delineate the components of decidua (if present), invasive trophoblast, and myometrium, respectively. The other immunohistochemistry stain which can be helpful in the setting of villous enlargement/irregularity is p57; in cases of complete hydatidiform mole, it demonstrates loss of staining in stromal cells and cytotrophoblast. However, while p57 is helpful in excluding complete hydatiform mole, it cannot distinguish partial hydatiform mole from hydropic change in non-molar pregnancy. ${ }^{5}$ For this scenario, ploidy studies can differentiate a partial hydatiform mole (triploid gestation) from non-molar causes of hydropic change (diploid). As mentioned above, the tissue analyzed in this

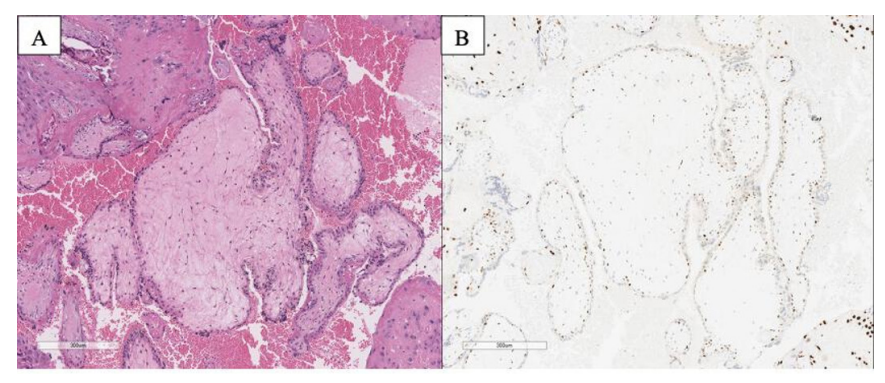

Figure 6 (A) High power hematoxylin and eosin image of enlarged villi with irregular contours and (B) corresponding p57 immunostain demonstrating intact staining of villous stromal cells and cytotrophoblast. case was unsuitable for ploidy analysis, but the overall index of suspicion for partial hydatiform mole was low.

The patient's post-operative recovery was unremarkable and her serum HCG returned to $<1 \mathrm{IU} / \mathrm{L} 2$ weeks following her surgery.

\section{DR BOUCHARD AND DR CLARK: HOW ARE NON-METASTATIC PLACENTAL SITE TROPHOBLASTIC TUMORS MANAGED?}

Placental site trophoblastic tumors are a rare entity, occurring in approximately 1 in 100000 pregnancies and there are less than 500 cases reported in the literature. ${ }^{6}$ There is a lack of robust prospective data to guide treatment planning. Based on the results of observational studies, patients with uterine confined disease can experience a high rate of cure with hysterectomy. ${ }^{67}$ Rate of lymph node metastasis is 5-6\% and some centers are recommending routine lymphadenectomy; however, it is not routine practice in our center to remove normal appearing pelvic and para-aortic lymph nodes in this patient population. ${ }^{7}$

One of the largest series to date is the analysis of 108 cases by Zhao et al in China. ${ }^{8}$ In their cohort, 71 cases were stage I disease, all of whom were treated with surgery and 49/71 received adjuvant chemotherapy with a mean survival of 171.3 months (95\% Cl 166 to 176.6). Chemotherapy in stage I patients was not a significant factor influencing both overall survival and recurrence rates $(p=0.799)$. On univariate analysis stage, interval between antecedent pregnancy and placental-site trophoblastic tumors (cutoff of 36 months), prognosis score and necrosis were all significant for overall survival and recurrence; however on multivariate analysis only stage was an independent risk factor for survival (OR 3.5, 95\% Cl 1.105 to 11.679 ) and relapse (OR 5.7, 95\% Cl 1.3 to 24.5$){ }^{8}$

\section{What are the treatment options for a woman who desires to preserve fertility?}

As mentioned, there is a lack of high-quality data regarding the overall management of placental site trophoblastic tumors, making nuanced situations like fertility sparing even more challenging. A summary of the literature can be found in Table 1. Most patients will undergo either hysteroscopic or transuterine resection of the involved area followed by chemotherapy. Saso et al reported a series of five women who underwent a modified Strassman procedure with lymph node dissection. This procedure involves the temporary occlusion of uterine vasculature through the use of vascular slings or bulldog clamps incorporating the uterine artery near its origin at the internal iliac artery followed by resection of the involved area. Unfortunately, 4/5 women had unclear or positive surgical margins and went on to have completion hysterectomy, two of whom had residual placental site trophoblastic tumor on final specimen..$^{9}$ In a series of six women with a mix of stage I-III disease, five underwent a partial resection, all received chemotherapy, and at a mean follow-up of 45 months (10-104), all women were without evidence of disease recurrence. ${ }^{10}$ of the 37 patients reviewed, there was only one death, as almost all failures of fertility sparing treatment were able to be salvaged with either radical surgery, additional chemotherapy or a combination of both. Patients and physicians should be cautious in interpreting these findings and remain aware that follow-up, outcomes, and regimens were highly variable in these series. Fertility sparing surgery is not the standard of care in the management of placental site trophoblastic tumor but could be 
Table 1 Summary of fertility sparing reports in the literature

\begin{tabular}{|c|c|c|c|c|c|c|}
\hline Author & $\begin{array}{l}\text { No. of } \\
\text { patients }\end{array}$ & Stage & Procedure(s) & Chemotherapy & Outcomes & $\begin{array}{l}\text { Follow-up } \\
\text { (months) }\end{array}$ \\
\hline Shen $^{10}$ & 6 & $\begin{array}{l}\text { I-5 } \\
\text { III-1 }\end{array}$ & $\begin{array}{l}\text { Open uterine resection } \\
\text { Hysteroscopy }^{2}\end{array}$ & Yes $^{11}$ & $\mathrm{NED}^{11}$ & $47(10,104)$ \\
\hline Saso $^{9}$ & 5 & - & $\begin{array}{l}\text { Partial uterine resection + } \\
\text { LND }\end{array}$ & $\begin{array}{l}\text { Yes }^{5} \\
\mathrm{No}^{1}\end{array}$ & $\begin{array}{l}\text { Recurrence } \\
\text { NED }^{1}\end{array}$ & - \\
\hline Pfeffer ${ }^{15}$ & 1 & I & Wedge resection & Yes & Recurrence, 3 months & 30 \\
\hline Tsuji ${ }^{17}$ & 1 & I & Wedge resection & No & NED & 9 \\
\hline Zhao $^{8}$ & 23 & $\begin{array}{l}\mathrm{I}-15 \\
\mathrm{III}-8\end{array}$ & $\begin{array}{l}\text { D\&C } \\
\text { Wedge resection } \\
\text { Pulmonary lobectomy }\end{array}$ & Yes (20) & $\begin{array}{l}\text { NED (22) } \\
\text { Death }^{1}\end{array}$ & $25(8,137)$ \\
\hline
\end{tabular}

D\&C, dilation and curettage; LND, lymph node dissection; NED, no evidence of disease.

considered in highly selected patients who are appropriately counseled with experienced surgeons in high-volume centers.

\section{DR BOUCHARD AND DR CLARK Closing summary}

Placental site trophoblastic tumors represent a rare but potentially curable sub-type of gestational trophoblastic neoplasia when diagnosed early. This case highlights the challenges in accurate diagnosis of these lesions pre-operatively in order to appropriately counsel patients on management strategies, especially in younger women who wish to retain fertility. As discussed, there are no welldefined radiographic characteristics that can help in distinguishing placental site trophoblastic tumor from other uterine lesions like placenta increta or arteriovenous malformation. As is typical with other rare diseases, there are no high-quality data to guide recommendations for standard of care treatment, and certainly even less information pertaining to uterine sparing procedures. The studies outlined in this case report show that fertility sparing options can range from a hysteroscopic resection to open uterine wedge resection and demonstrate that nearly 2/3 cases result in cure, although with variable lengths of follow-up (Table 1). The use of post-operative chemotherapy is almost universal following fertility sparing procedures, whereas in cases of definitive hysterectomy for stage I disease it is either omitted or offered based on the classic high-risk features of size $>1 \mathrm{~cm},>5$ mitoses/high-power field, age over 34 years old, and time since antecedent pregnancy $>4$ years. ${ }^{11-13}$ A recent report by Ghorani et al ${ }^{14}$ demonstrated the activity of pembrolizumab in the treatment of four cases of drug resistant gestational trophoblastic neoplasia, including two cases of placental-site trophoblastic tumors that over-expressed the programmed-death ligand, PDL-1. Like in other solid tumors, this is an exciting move forward towards new agents that show durable response with minimal toxicity in clinical situations thought previously to be incurable.

In patients with a strong desire for future fertility, with an understanding of the risks associated with this approach and the likely need for post-operative platinum-etoposide based chemotherapy, it could be considered by an expert team. Unless more sensitive and specific tools for pre-operative diagnosis of placental site trophoblastic tumor are developed, clinicians who encounter a patient similar to ours must consider the possibility that a uterine mass with these characteristics, in this clinical context, may be an intermediate trophoblastic lesion.

Contributors GB-F and MC contributed to design, data collection, analysis and manuscript writing. NT, BC and UM contributed to data collection, analysis and manuscript writing.

Funding The authors have not declared a specific grant for this research from any funding agency in the public, commercial or not-for-profit sectors.

Competing interests None declared.

Patient consent for publication Not required.

Provenance and peer review Commissioned; internally peer reviewed.

\section{REFERENCES}

1 Huang MW, Muradali D, Thurston WA, et al. Uterine arteriovenous malformations: gray-scale and Doppler US features with MR imaging correlation. Radiology 1998;206:115-23.

2 Tanaka YO, Shigemitsu S, Ichikawa Y, et al. Postpartum MR diagnosis of retained placenta accreta. Eur Radiol 2004;14:945-52.

3 Bartels HC, Postle JD, Downey P, et al. Placenta accreta spectrum: a review of pathology, molecular biology, and biomarkers. Dis Markers 2018;2018:1-11.

4 Jauniaux E, Hussein AM, Zosmer N, et al. A new methodologic approach for clinico-pathologic correlations in invasive placenta previa accreta. Am J Obstet Gynecol 2020;222:379.e1-379.e11.

5 Banet N, DeScipio C, Murphy KM, et al. Characteristics of hydatidiform moles: analysis of a prospective series with p57 immunohistochemistry and molecular genotyping. Mod Pathol 2014;27:238-54.

6 Goldstein DP, Berkowitz RS. Current management of gestational trophoblastic neoplasia. Hematol Oncol Clin North Am 2012;26:111-31.

7 Davis MR, Howitt BE, Quade BJ, et al. Epithelioid trophoblastic tumor: a single institution case series at the New England Trophoblastic Disease Center. Gynecol Oncol 2015;137:456-61.

8 Zhao J, Lv WG, Feng FZ, et al. Placental site trophoblastic tumor: a review of 108 cases and their implications for prognosis and treatment. Gynecol Oncol 2016;142:102-8.

9 Saso S, Haddad J, Ellis P, et al. Placental site trophoblastic tumours and the concept of fertility preservation. BJOG 2012;119:369-74. discussion 374.

10 Shen X, Xiang Y, Guo L, et al. Fertility-preserving treatment in young patients with placental site trophoblastic tumors. Int J Gynecol Cancer 2012;22:869-74.

11 Horowitz NS, Goldstein DP, Berkowitz RS. Placental site trophoblastic tumors and epithelioid trophoblastic tumors: biology, natural history, and treatment modalities. Gynecol Oncol 2017;144:208-14.

12 Froeling FEM, Ramaswami R, Papanastasopoulos P, et al. Intensified therapies improve survival and identification of novel prognostic 


\section{Case study}

factors for placental-site and epithelioid trophoblastic tumours. $\mathrm{Br} \mathrm{J}$ Cancer 2019;120:587-94.

13 Hancock B, Froeling FEM, Ramaswami R. The ISSTD global placental site and epithelioid trophoblastic tumor (PSTT/ETT) database - an analysis of 326 patients. ISSTD XVIII World Congress on Gestational Trophoblastic Disease. Bali, Indonesia 2015.

14 Ghorani E, Kaur B, Fisher RA, et al. Pembrolizumab is effective for drug-resistant gestational trophoblastic neoplasia. Lancet 2017;390:2343-5.
15 Pfeffer PE, Sebire N, Lindsay I, et al. Fertility-sparing partial hysterectomy for placental-site trophoblastic tumour. Lancet Oncol 2007;8:744-6.

16 Machtinger R, Gotlieb WH, Korach J, et al. Placental site trophoblastic tumor: outcome of five cases including fertility preserving management. Gynecol Oncol 2005;96:56-61.

17 Tsuji Y, Tsubamoto H, Hori M, et al. Case of PSTT treated with chemotherapy followed by open uterine tumor resection to preserve fertility. Gynecol Oncol 2002;87:303-7. 